\title{
Identification of the Characteristic Volatile Flavor Compounds Formed by Cooking Squid (Todarodes pacificus STEENSTRUP)
}

\author{
Kikue Kubota, Yukiko Matsukage, ${ }^{*}$ Youko Sekiwa and Akio Kobayashi \\ Laboratory of Food Chemistry, Department of Nutrition and Food Science, Ochanomizu University, Tokyo 112, Japan
}

Received December 8, 1995

\begin{abstract}
Volatile compounds were isolated from cooked squid (Todarodes pacificus STEENSTRUP) using a porous polymer resin with Tenax TA column chromatography. Aroma extract dilution analysis determined the following six compounds as the main potent odorants of cooked squid: 4,5-dimethylthiazole (green), 2-acetyl-2-thiazoline (nutty), 2,5-dimethylpyrazine (popcorn-like), methional (potato, soy sauce), furaneol (caramel-like) and an unidentified compound (floral). Among them, based on the high concentration and odor characteristics, it is concluded that furaneol was the most important compound contributing to the sweet aroma of cooked squid.
\end{abstract}

Keywords: squid, cooked squid odor, potent odorant, furaneol, Tenax TA adsorption

Squid is one of the most popular seafoods consumed in Japan. A large number of species of squid are known throughout the world, and the three main species, i.e., Sepia esculenta HOYLE, Doryteuthis bleekeri (KEFERSTEIN) and Todarodes pacificus STEENSTRUP are widely used for processed food in Japan. Some of them are eaten raw as sashimi, but most of them are consumed after cooking or other processing. Todarodes pacificus STEENSTRUP (surume ika in Japanese) is caught in large quantities around the Japan coast and is known to be good for cooking. Uncooked squid possesses only a weak odor, while a strong and characteristic odor, which is quite different from that of other fish, is generated by cooking. The odor compounds in the head-space vapor of roasted squid (Kasahara \& Nishibori, 1985) and the volatile compounds of cooked squid by simultaneously using steam distillation and extraction (Lee et al., 1989; Koizumi et al., 1990) have been analyzed and more than 75 volatiles were identified. However, the significant aroma compounds have not been found yet. Recent flavor research has stressed the importance of investigating the active odor compound, and in the last decade, several combinations of gas chromatography and column effluent sniffing techniques (aroma extract dilution analysis, AEDA) have been used to determine the active odor compounds in the food volatiles (Acree et al., 1984; Schieberle \& Grosch, 1987; Chung \& Cadwallader, 1994). In our preliminary study, the aroma concentrate prepared by simultaneous distillation and extraction or by steam distillation under reduced pressure did not sufficiently present the characteristic odor of cooked squid. A method for concentrating the volatile compounds in water into a column packed with a porous polymer resin has recently been applied for food flavor research, and this procedure was very effective for preparing a volatile concentrate which retained the characteristic sweet odor of cooked squid. Squid is cooked in various ways like baking, boiling for salad and other dishes,

\footnotetext{
* Present address: Asashi Brewery Co., Ltd
}

pan-frying, tempura and others. But, since raw squid contains about $80 \%$ moisture, it is expected that the mantle muscle except the skin is usually heated with steam and kept under $100^{\circ} \mathrm{C}$ during cooking. In the present study, the characteristic odor components of squid cooked in a small amount of boiling water were investigated in further detail using this porous polymer resin, and some potent odor compounds of cooked squid were then determined using gas chromatography (GC), mass spectrometry (MS), and an aroma extract dilution analysis (AEDA).

\section{Materials and Methods}

Materials Squid (Todarodes pacificus STEENSTRUP) frozen on the ship just after catching were purchased from a wholesaler (Sakaiminato, Tottori prefecture, Japan). The average weight was almost $450 \mathrm{~g}$.

Almost all the standard compounds were commercially available. 2,5-Dimethyl-4-hydroxy-3(2H)-furanone and 5methyl-4-hydroxy-3(2H)-furanone were donated from $\mathrm{T}$. Hasegawa Co., Ltd., Tokyo, while the pyrazines and 2-acetyl2-thiazoline were donated from Ogawa \& Co., Ltd., Tokyo, and Takasago Co., Ltd., Tokyo, respectively.

Isolation of the volatile compounds After the frozen squid had been thawed out in the package under running water, the mantle muscle with skin $(1600 \mathrm{~g})$ was cut into $5-\mathrm{mm}$ squares and boiled in purified water (60\% weight of the squid) for $30 \mathrm{~min}$. Filtration through three layers of cotton gauze and centrifugation for $10 \mathrm{~min}$ at $3000 \mathrm{rpm}$ removed the solids, and the obtained soup was passed through a $1.7 \mathrm{~cm}$ i.d. $\times 20 \mathrm{~cm}$ bed-height column packed with preconditioned Tenax TA resin in methanol (80-100 mesh). This was eluted with $150 \mathrm{ml}$ of purified water to remove the non-volatile water-soluble substances like sugars and amino acids. The volatiles were then desorbed from the resin with $100 \mathrm{ml}$ of ethyl ether. After being dried over anhydrous sodium sulfate, the solvent of the ethereal extracts was distilled off at $39^{\circ} \mathrm{C}$ to obtain the volatiles. The Tenax TA column was regenerated by succes- 
sive washing with methanol, $1 \% \mathrm{NaOH}$, water, $1 \% \mathrm{HCl}$, water, methanol, ethyl ether, and methanol. When Porapak Q was used instead of Tenax TA as the polymer resin, ethyl benzene homologs and acetophenone-related compounds, the degradation products of the resin, tended to disturb the main GC peaks of the squid volatile concentrate. Tenax TA was therefore used for the adsorption of the volatile squid compounds. The collected volatiles were fractionated into basic, acid and neutral fractions in the column by successively eluting with $1 \% \mathrm{HCl}, 1 \% \mathrm{NaOH}$, and ethyl ether (Shimoda et al., 1987).

Gas chromatography (GC) A Shimadzu Model 7A gas chromatograph equipped with a flame ionization detector was used for the GC analysis. A fused silica open tubular column $(50 \mathrm{~m} \times 0.25 \mathrm{~mm}$ i.d. $)$, which had been coated with PEG 20M (Gasukuro Kogyo) was set up. The oven temperature was programmed from $60^{\circ} \mathrm{C}$ to $180^{\circ} \mathrm{C}$ at $2^{\circ} \mathrm{C} / \mathrm{min}$, and the injector and detector temperatures were maintained at $200^{\circ} \mathrm{C}$. Nitrogen gas was used as the carrier at a flow rate of $1.0 \mathrm{ml} /$ min. A Hewlett-Packard 5890 Series II gas chromatograph equipped with TCD was used for the sniffing analysis, while the other conditions were almost the same as those just described, except for using helium as the carrier gas.

GC-MS and GC-FT-IR analyses of the volatiles GC-MS spectra were recorded using a JEOL Model DX-300 mass spectrometer, which was combined with a HewlettPackard Model 5790A gas chromatograph. The gas chromatographic conditions were the same as those just described under an ionization voltage of $70 \mathrm{eV}$ and ion source temperature of $200^{\circ} \mathrm{C}$. The structures of the acetyl pyrazines and $N, N$-dimethylthioformamide were confirmed by their infrared spectra which were recorded using a Perkin-Elmer 8500 GC-FT-IR instrument. GC conditions were the same as those used for the GC-MS, except for using a $0.32 \mathrm{~mm}$ i.d.X $50 \mathrm{~m}$ PEG 20M FSWCOT column.

Determination of the potent odor compounds To determine the potent odorants in the cooked squid volatiles, the GC eluate sniffing and dilution method (AEDA) of Shieberle \& Grosch (1987) was applied. After stepwise dilution of the concentrate by adding diethyl ether, GC and GC-sniffing were performed for each diluted sample. The significant odorous peaks in the eluate were determined by evaluating the odor and dilution values (Shieberle \& Grosch, 1987).

\section{Results and Discussion}

Yield and identification of the volatiles The yield of the volatile concentrate from cooked squid was approximately $0.18 \mathrm{mg} / 100 \mathrm{~g}$, calculated on the basis of the wet weight of the mantle muscle. The concentrate had a sweet and fishy odor strongly characteristic of cooked squid. The volatile concentrate could not be clearly fractionated into its basic, acid and neutral fractions, because both main components of N,N-dimethylthioformamide and 3-methylthiopropanol (methionol) were included in each fraction. However, some new compounds were identified by fractionation and confirmed the results of the previous report (Koizumi et al., 1990) that the characteristic odor of cooked squid was best reproduced in the neutral fraction. Almost all the major constituents were identified by comparing their mass spectra and retention indices from GC with those of the reference compounds and are listed in Table 1. The concentration of each compound was calculated from the yield of the aroma concentrate and the peak area \% by GC. Since compounds 24 and 25 could not be clearly separated by GC and showed very similar mass spectra, except for their molecular ions $(\mathrm{m} / z 136$ and 122), they were identified as 2-acetyl-3-methylpyrazine and 2-acetylpyrazine, respectively, using mass chromatography and comparing their KI values with those of authentic samples. It was determined that compound 33 was $N, N$ dimethylthioformamide, because the absorption by FT-IR at $1137 \mathrm{~cm}^{-1}$ showed the existence of a thiocarbonyl group in the molecule, and MS spectrum $[m / z(\%): 91(4, \mathrm{M}+2), 89$ $\left.\left(100, \mathrm{M}^{+}\right), 74(12), 44(67), 42(42), 30(38)\right]$ and $\mathrm{KI}$ value by $\mathrm{GC}$ well coincided with those of an authentic sample. In this study, 54 compounds were identified. The main components making up more than $3 \%$ of the peak area by GC (5.4 ppm concentration) were the following: 3-pentanone, 3-hexanone, 3-methylthiopropanol (methionol), N,N-dimethylthioformamide, 2,5-dimethyl-4-hydroxy-3(2H)-furanone (furaneol) and 5-methyl-4-hydroxy-3(2H)-furanone. Forty-one constituents, including the foregoing six main components were newly identified as volatile components of the cooked squid. Among them, heterocyclic compounds including hydroxy furanones, pyridines, pyrazines, pyrroles, thiazoles, and thiazoline are known to be the major cooked flavor compounds produced in the Maillard reactions and many related compounds were identified in the present study. $N, N$ dimethylformamide was also found in the flavor components of cooked egg (Umano et al., 1990), roasted dried squid (Kawai et al., 1991), and blue crab meat (Chung \& Cadwallader, 1993). On the other hand, tetramethyl thiourea was found only in the volatile fraction of Parmesan cheese (Barbieri et al., 1994), and N,N-dimethylthioformamide has not been identified as a flavor constituents. It is considered that these two sulfur-containing compounds are not the flavor substances produced in squid, but the metabolites or originate from the feed, though their actual origin is unknown. It is also known that furaneol possesses a caramel-like flavor and is a potent odorant of strawberry (Büchi et al., 1973) and pineapple (Rodin et al., 1965), as well as being one of the typical roasted odor compounds (Shaw \& Berry, 1977). Therefore, it was postulated that furaneol would contribute the sweet characteristic odor of cooked squid. To investigate in detail which components would mainly contribute to the characteristic odor of cooked squid, an aroma extract dilution analysis was carried out.

Evaluation of the potent odorants To determine some of the potent odorants, double dilution of the volatile concentrate with diethyl ether was carried out 4 times. Among the main constituents, 3-pentanone and 3-hexanone could not reproduce their own odor. The sweet and medicine-like odor of 5-methyl-4-hydroxy-3(2H)-furanone was perceived within the double dilution, and the fermented cheese-like odor of methionol and mold-like odor of $N, N$-dimethylthioformamide were found within the 4-fold dilution. 2-Acetylpyrazine and 3-methyl-2-acetylpyrazine, and maltol showed a popcorn-like and sweet odor, respectively, within the eight-fold 
Table 1. Volatile compounds identified in cooked squid mantle muscle.

\begin{tabular}{|c|c|c|c|}
\hline No. & Compound & $\mathrm{RI}^{\mathrm{b}}$ & Concentration $(\mathrm{ppm})^{\mathrm{c}}$ \\
\hline 1 & ethyl formate & 820 & 0.14 \\
\hline 2 & ethyl acetate & 878 & 0.14 \\
\hline 3 & ethanol & 921 & 3.18 \\
\hline 4 & 3-pentanone & 964 & 11.07 \\
\hline 5 & 3-hexanone & 985 & 15.71 \\
\hline 6 & methyl 2-methylbutanoate & 999 & 2.03 \\
\hline 7 & 2-propylfuran & 1124 & I. .42 \\
\hline 8 & pyridine $^{a)}$ & 1159 & 0.29 \\
\hline 9 & 2-methylpyridine ${ }^{a)}$ & 1182 & 0.04 \\
\hline 10 & 2-methylpyrazine ${ }^{a)}$ & 1238 & 0.14 \\
\hline 11 & 3-hydroxy-2-butanone & 1256 & 1.46 \\
\hline 12 & 3-methylpyridine & 1264 & 0.13 \\
\hline 13 & $N, N$-dimethylformamide & 1295 & 0.59 \\
\hline 14 & 2,5-dimethylpyrazine ${ }^{a)}$ & 1297 & 0.76 \\
\hline 15 & 2,3-dimethylpyrazine ${ }^{a)}$ & 1314 & 0.54 \\
\hline 16 & 4,5-dimethylthiazole & 1341 & 0.50 \\
\hline 17 & trimethylpyrazine $^{a)}$ & 1371 & 0.65 \\
\hline 18 & acetic acid & 1415 & 2.81 \\
\hline 19 & 3-methylthiopropanal & 1421 & 0.31 \\
\hline 20 & 2-furancarbaldehyde & 1425 & 0.81 \\
\hline 21 & 2-ethylhexanol & 1461 & 0.27 \\
\hline 22 & $1 H$-pyrrole & 1477 & 1.03 \\
\hline 23 & benzaldehyde ${ }^{a)}$ & 1479 & 0.47 \\
\hline 24 & 2-acetyl-3-methylpyrazine & 1578 & 3.29 \\
\hline 25 & 2-acetylpyrazine & 1578 & \\
\hline 26 & 2-acetylthiazole & 1597 & 1.76 \\
\hline 27 & 2-acetylpyridine & 1599 & $t^{(d)}$ \\
\hline 28 & 1-furylmethanol ${ }^{a)}$ & 1623 & 0.61 \\
\hline 29 & 3-methylbutanoic acid & 1636 & 0.83 \\
\hline 30 & $\begin{array}{l}\text { 3,4-dihydro-5-ethyl-2(3H)- } \\
\text { furanone }\end{array}$ & 1655 & 0.07 \\
\hline 31 & 3-methylthiopropanol & 1676 & 5.54 \\
\hline 32 & 2-acetyl-2-thiazoline & 1700 & 0.25 \\
\hline 33 & $\begin{array}{l}N, N- \\
\quad \text { dimethylthioformamide }\end{array}$ & 1764 & 6.88 \\
\hline 34 & hexanoic $\operatorname{acid}^{a)}$ & 1808 & 0.68 \\
\hline 35 & benzyl alcohol ${ }^{a)}$ & 1829 & 0.74 \\
\hline 36 & 2-phenylethanol ${ }^{a)}$ & 1861 & 0.16 \\
\hline 37 & tetramethyl thiourea & 1872 & 0.97 \\
\hline 38 & benzothiazole & 1903 & 0.11 \\
\hline 39 & maltol & 1909 & 3.37 \\
\hline 40 & 2-acetylpyrrole & 1915 & 0.79 \\
\hline 41 & dodecanol & 1919 & 1.00 \\
\hline 42 & phenol $^{a)}$ & 1958 & 0.72 \\
\hline 43 & I $H$-pyrrole-2-carbaldehyde & 1963 & 0.36 \\
\hline 44 & $\begin{array}{l}\text { 2,5-dimethyl-4-hydroxy- } \\
\text { 3(2H)-furanone } \\
\text { (furaneol) }\end{array}$ & 1988 & 10.49 \\
\hline 45 & $\begin{array}{l}(Z, Z, Z)-5,8,11 \text {-tetradeca- } \\
\quad \text { trien-2-one }\end{array}$ & 1999 & 1.12 \\
\hline 46 & octanoic acid ${ }^{a j}$ & 2022 & 2.75 \\
\hline 47 & $\begin{array}{l}\text { 5-methyl-4-hydroxy-3(2H)- } \\
\text { furanone }\end{array}$ & 2069 & 28.35 \\
\hline 48 & 2-phenoxyethanol & 2080 & 1.62 \\
\hline 49 & nonanoic acid & 2127 & 2.50 \\
\hline 50 & 2-amino-1-phenylethanone & 2156 & 1.08 \\
\hline 51 & methyl hexadecanoate & 2178 & $t^{d\rangle}$ \\
\hline 52 & $\begin{array}{l}\text { 5,6-dihydro-3,5-dihydroxy- } \\
\text { 2-methyl-4H-pyran-4- } \\
\text { one }\end{array}$ & 2209 & 4.57 \\
\hline 53 & decanoic acid & 2220 & 1.10 \\
\hline 54 & hexadecanol & 2343 & 4.64 \\
\hline
\end{tabular}

a) These compounds were already reported in the aroma constituent from cooked squid (Lee et al., 1989; Koizumi et al., 1990). Peak 17 was found in the roasted oval squid (Kasahara \& Nishibori, 1985).

b) Retention index: calculated Kovats index in a PEG $20 \mathrm{M}(0.25 \mathrm{~mm}$ i.d. $\times 50$ m) column.

c) Calculated from the yield of aroma concentrate and the peak area \% by GC. a) $<0.01 \mathrm{ppm}$.
Table 2. Potent odorants of cooked squid.

\begin{tabular}{lcl}
\hline \multicolumn{1}{c}{ Compound } & Dilution value $n^{\alpha}$ & Odor description \\
\hline 2,5-dimethylpyrazine & 4 & popcorn-like \\
2-acetyl-2-thiazoline & 4 & nutty \\
4,5-dimethylthiazole & 4 & green \\
3-methylthiopropanal & 4 & potato, soy sauce \\
2,5-dimethyl-4-hydroxy- & 4 & caramel-like \\
3(2H)-furanone (furaneol) & & \\
unknown (RI 2160) & 4 & floral \\
2-acetylpyrazine (and/or) & & \\
3-methyl-2-acetylpyrazine & 3 & popcorn-like \\
2-acetylthiazole & 3 & popcorn-like \\
benzothiazole & 3 & medicinal \\
maltol & 3 & sweet \\
\hline a) No. of dilutions $-2^{n}$ & &
\end{tabular}

dilution. Finally, in the 16-fold diluted concentrate, only six compounds were perceived as listed in Table 2 with their corresponding odor characteristics. These presented a popcorn or almond-like roasted flavor, sweet and caramel flavor, soy sauce, and floral or green-note flavor. Since, apart from green or floral note, these odors were generally perceived in cooked squid, it is considered that these compounds were the potent odorants of cooked squid. Almost all of these potent odorants, i.e., 4,5-dimethylthiazole, 2-acetyl-2-thiazoline, 2-acetylpyrazine, maltol, furaneol already mentioned and 3-methylthiopropanal (methional), were identified for the first time in this study as the volatile components of cooked squid.

Recovery of furaneol Furaneol is easily dissolved in water because of its polarity. However, this compound is also known to be somewhat unstable ( Hirvi et al., 1980). In our preliminary experiment, furaneol was not detected in the odor concentrate that had been prepared by steam distillation. Therefore, the recovery of furaneol during the isolation procedure was investigated. Cooked squid soup $(800 \mathrm{ml})$ including $9.0 \times 10^{-2} \mathrm{mg}$ of authentic furaneol (almost the same as the amount in the squid aroma concentrate) was prepared from one $\mathrm{kg}$ of squid mantle muscle. One-half was submitted to Tenax TA column chromatography in the same way as that already described to recover the volatiles. The other half was submitted to steam distillation under reduced pressure $\left(40^{\circ} \mathrm{C}, 20\right.$ Torr) with a rotary evaporator, and the volatiles were extracted with ethyl ether from the distillate. After adding $5 \times 10^{-3} \mathrm{mg}$ of methyl decanoate as the internal standard (i.s.) to both ethyl ether extracts, the solvent was distilled off to obtain the odor concentrate. GC analyses from the peak area ratio against i.s. showed that furaneol was recovered at $118 \%$ and $3.6 \%$ of i.s. by the Tenax TA and steam distillation procedures, respectively; i.e., little furaneol was recovered by steam distillation. This result well explains the fact that the steam distillate of cooked squid did not sufficiently reveal the true flavor. This result and the organoleptic characteristics enable us to conclude that furaneol was the most important potent constituent of the sweet odor formed in squid by cooking. The process for the formation of furaneol in squid during cooking will be described elsewhere.

\section{References}

Acree, T.E., Barnard, J. and Cunningham, D.G. (1984). The analysis of odor-active volatiles in gas chromatographic effluents. In "Analy- 
sis of Volatiles: Methods and Applications," ed. by P. Schreier. Walter de Gruyter, Berlin, pp. 251-267.

Barbieri, G., Bolzoni, L., Careri, M., Mangia, A., Parolari, G., Spagnoli, S. and Virgili, R. (1994). Study of the volatile fraction of Parmesan cheese. J. Agric. Food Chem., 42, 1170-1176.

Büchi, G., Demole, E. and Thomas, A.F. (1973). Syntheses of 2, 5-dimethyl-4-hydroxy-2,3-dihydrofuran-3-one (furaneol), a flavor principle of pineapple and strawberry. J. Org. Chem., 38, 123-125.

Chung, H.Y. and Cadwallader, K.R. (1993). Volatile components in blue crab (Callinectes sapidus) meat and processing byproduct. $J$. Food Sci., 58, 1203-1207.

Chung, H.Y. and Cadwallader, K.R. (1994). Aroma extract dilution analysis of cooked crab claw meat volatiles. J. Agric. Food Chem., 42, 2867-2870.

Hirvi, T., Honkanen, E. and Pyysalo, T. (1980). Stability of 2,5dimethyl-4-hydroxy-3(2H)-furanone and 2,5-dimethyl-4-methoxy$3(2 \mathrm{H})$-furanone in aqueous buffer solutions. Food Sci. Technol., 13, 324-325.

Kawai, T., Ishida, Y., Kakiuchi, H., Ikeda, N., Higashida, T. and Nakamura, S. (1991). Flavor components of dried squid. J. Agric. Food Chem., 39, 770-777.

Kasahara, K. and Nishibori, K. (1985). Volatile components of roasted fishes. Nippon Suisan Gakkaishi, 51, 489-492.

Koizumi, C., Ohshima, T. and Lee, E.-H. (1990). Volatile constituents of processed squid product. J. Korean Soc. Food Nutr., 19, 547-554.

Lee, E.-H., Choi, B.-D., Lee, K.-H. and Ryu, H.-S. (1989). Flavor components in the squid processing. Bull. Korean Fish. Soc., 22, 370-374.

Rodin, J.O., Himel, C.M., Silverstein, R.M., Leeper, R.W. and Gortner, W.A. (1965). Volatile flavor and aroma components of pineapple. $J$. Food Sci., 30, 280-285.

Shaw, P.E. and Berry, R.E. (1977). Hexose-amino acid degradation studies involving formation of pyrroles, furans and other low molecular weight products. J. Agric. Food Chem., 25, 641-644.

Schieberle, P. and Grosch, W. (1987). Evaluation of the flavour of wheat and rye bread crusts by aroma extract dilution analysis. $Z$. Lebensm. Unters. Forsch., 185, 111-113.

Shimoda, M., Hirano, K. and Osajima, Y. (1987). Concentration of volatile components in foods with porous polymer column. Bunseki Kagaku, 36, 792-798.

Umano, K., Hagi, Y., Shoji, A. and Shibamoto, T. (1990). Volatile compounds formed from cooked whole egg, egg yolk, and egg white. J. Agric. Food Chem., 38, 461-464. 